\title{
Síndrome de Stewart-Treves: Caso clínico
}

\author{
RICARDO SILVARIÑO, VALENTINA MÉROLA, LUIS FÍGOLI ${ }^{\mathrm{a}}$, \\ CECILIA ROMERO, JIMENA LAPIEDRA, JUAN ALONSO
}

\section{Stewart-Treves syndrome. Report of one case}

\begin{abstract}
Angiosarcomas are malignant tumors derived from the endothelium of blood vessels (hemangiosarcomas) or lymph vessels (lymphangiosarcomas). Lymphedema of the limbs is considered secondary when extrinsic injuries are observed and primary when these injuries are not present. Stewart-Treves syndrome or a Lymphangiosarcoma, developed over a chronic lymphedema, is a rare complication described in mastectomized patients but it can be observed in lymphedemas located elsewhere. It appears as nodular skin lesions that grow, multiply quickly and frequently metastasize. We report a 40-year-old male with an angiosarcoma associated with primary chronic lower limb lymphedema. The patient consulted for a history of weight loss and malaise and appearance of violaceous lesions over the zone of lymphedema and inguinal lymph node involvement. A CT scan showed bilateral lung lesions and enlargement of inguinal and iliac lymph nodes. A biopsy of one of the skin lesions disclosed an angiosarcoma, diagnosis that was confirmed with immunohistochemistry. Chemotherapy was started but the patient died five months after the diagnosis.

(Rev Med Chile 2010; 138: 73-76).
\end{abstract}

Key words: Hemangiosarmoma; Lymphangiosarcoma; Lymphedema.

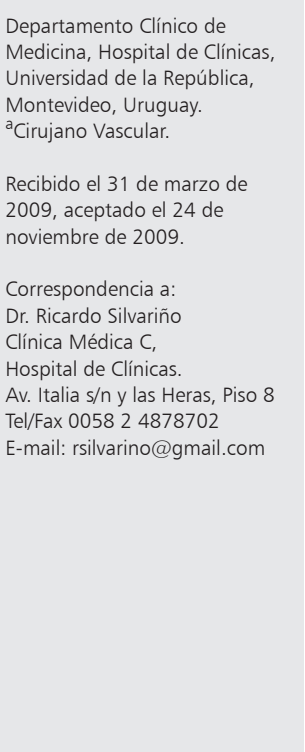

Departamento Clínico de Medicina, Hospital de Clínicas, Montevideo, Uruguay. ${ }^{a}$ Cirujano Vascular.

Recibido el 31 de marzo de 2009, aceptado el 24 de noviembre de 2009.

Av. Italia s/n y las Heras, Piso 8 Tel/Fax 005824878702

E-mail: rsilvarino@gmail.com
E 1 angiosarcoma es un tumor maligno derivado del endotelio que puede manifestarse en cualquier sitio anatómico, incluida la piel ${ }^{1}$. Según deriven del endotelio de vasos sanguíneos o linfáticos, se denominan hemangiosarcomas o linfangiosarcomas respectivamente. Los angiosarcomas asociados a linfedema crónico de extremidades son morfológicamente similares a otros angiosarcomas cutáneos ${ }^{2}$. El linfedema de miembros se denomina secundario cuando ocurre en el contexto de insuficiencia venosa crónica, filariasis, infecciones recurrentes, cirugía que compromete el flujo linfático, radioterapia o traumatismos del miembro. En ausencia de lesiones extrínsecas se denomina primario y se clasifica en congénito (habitualmente manifiesto antes del año de edad), precoz (presente antes de los 35 años) y tardío (en mayores de 35 años) ${ }^{3}$. El linfangiosarcoma desarrollado sobre un linfedema crónico (síndrome de Stewart-Treves) es una complicación descrita en 0,07 a $0,45 \%$ de pacientes que sobreviven más de cinco años a una mastectomía $a^{4,9}$. Si bien fue descrito como complicación en miembros superiores postmastectomía, el término se hizo extensivo a otras localizaciones menos frecuentes como miembros inferiores ${ }^{5}$. Se presenta como lesiones cutáneas nodulares que crecen, se multiplican con rapidez y metastatizan frecuentemente a pulmones y cavidad pleural ${ }^{6}$. La literatura registra aproximadamente 400 casos de angiosarcoma asociado a linfedema crónico, de los cuales la casi totalidad ocurrieron luego de mastectomía ipsilateral ${ }^{7}$. Se presenta un caso clínico de angiosarcoma vinculado a linfedema primario crónico de miembro inferior y se revisa la literatura a propósito del caso.

\section{Caso Clínico}

Paciente de 40 años, sexo masculino, procedente de medio urbano. Portador de linfedema 
primario tardío de miembro inferior derecho de tres años de evolución, valorado con linfocentellografia que había evidenciado ausencia de drenaje linfático en dicho miembro, tomografía computada (TC) de abdomen, pelvis y muslo sin alteraciones, y eco-Doppler arterial y venoso normales. Infecciones de piel recurrentes y de evolución tórpida en dicho miembro.

Consultó por cuadro de 1 mes y medio de evolución caracterizado por marcada astenia, anorexia, adelgazamiento de 10 kilos, con fiebre de hasta $38^{\circ} \mathrm{C}$ axilar. En la evolución se agregó tos con expectoración hemoptoica. Relataba lesiones sobre linfedema de reciente aparición. Del examen de ingreso destacaba: severo adelgazamiento, sin palidez ni fiebre. En miembro inferior derecho presentaba linfedema hasta raíz de muslo (Figura 1A) con múltiples lesiones nodulares de $5 \mathrm{~mm}$ de diámetro, firmes, violáceas, algunas descamadas y otras ulceradas con mayor concentración de lesiones en región poplítea (Figura 1B). En muslo proximal presentaba lesión, pediculada y ulcerada (Figura 1C). Lesión traumática de pie en vías de cicatrización. Adenomegalias inguinales a derecha, firmes, de $2 \mathrm{~cm}$ de diámetro que no formaban conglomerados. A nivel pleuropulmonar destacaba la presencia de ruidos subcrepitantes bibasales escasos. El resto del examen físico era normal. De la analítica de laboratorio destacaba hemograma, glicemia, función renal y pruebas hepáticas normales, VHS de $50 \mathrm{~mm}$ en la primera hora, y proteína C reactiva de $30 \mathrm{mg} / \mathrm{dL}$. Serología para VIH negativa. Baciloscopías y prueba de Mantoux negativos. La radiografía de tórax mostraba imagen nodular paracardíaca izquierda (Figura 1D). La TC de tórax, abdomen y pelvis mostraba múltiples nódulos pulmonares bilaterales a predominio bibasal (Figura 1E), mal delimitados y adenomegalias
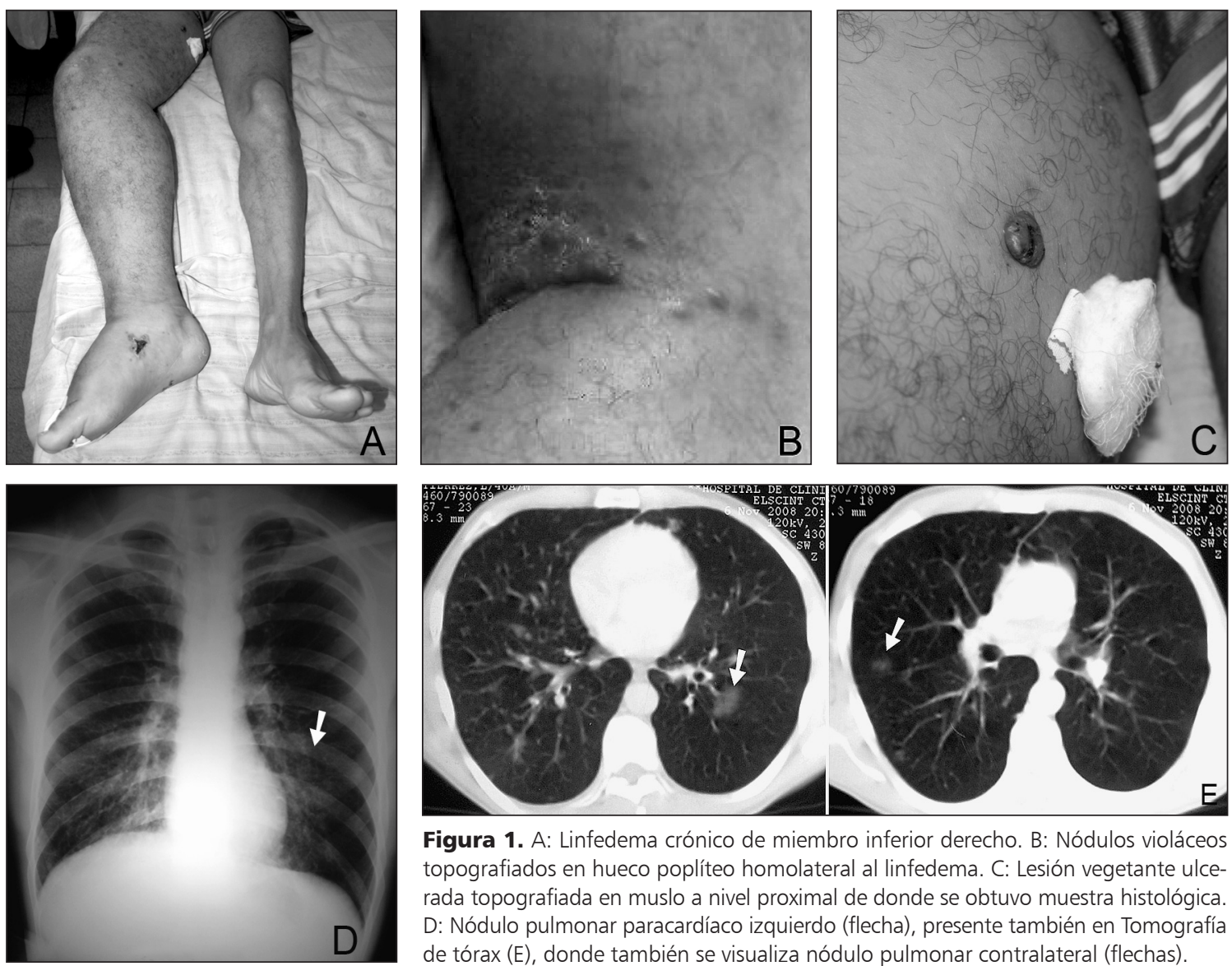

Figura 1. A: Linfedema crónico de miembro inferior derecho. B: Nódulos violáceos topografiados en hueco poplíteo homolateral al linfedema. C: Lesión vegetante ulcerada topografiada en muslo a nivel proximal de donde se obtuvo muestra histológica. D: Nódulo pulmonar paracardíaco izquierdo (flecha), presente también en Tomografía de tórax (E), donde también se visualiza nódulo pulmonar contralateral (flechas). 

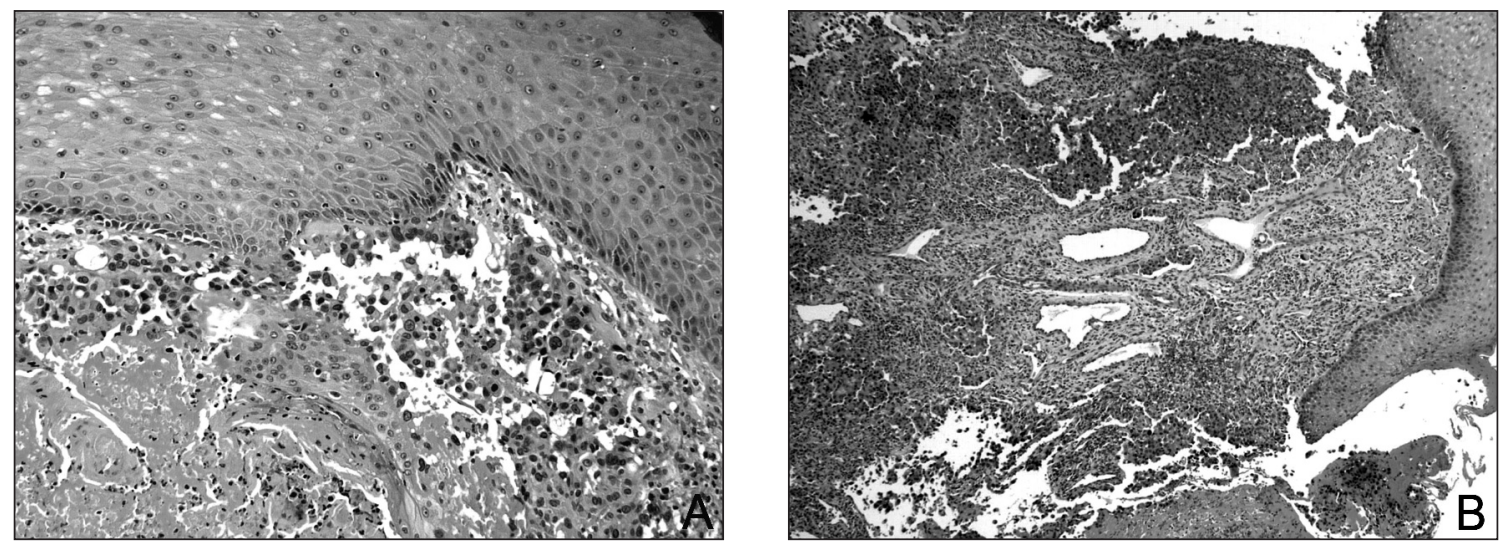

Figura 2. A: Infiltración dermo-hipodérmica por células fusiformes que envuelven espacios vasculares. B: Dilatación irregular de espacios vasculares revestidos por células poligonales con núcleos hipercoloreados. Infiltración por células fusiformes que deforman los espacios vasculares.

inguinales e iliacas externas derechas. Se realizó una biopsia incisional de lesión pediculada del muslo que fue compatible con angiosarcoma, con inmunohistoquímica concordante (positividad para marcadores de células endoteliales CD31 y CD34, el primero con elevada sensibilidad y especificidad $^{12,13}$ ) (Figura 2). Valorado por servicio de oncología clínica se inicio plan de poliquimioterapia. El paciente falleció a 5 meses del diagnóstico.

\section{Discusión}

Es extremadamente inhabitual el desarrollo de un angiosarcoma sobre un linfedema primario. Cuando ocurre en el contexto de una mastectomía se estima que el vaciamiento ganglionar axilar y la radioterapia local son factores precipitantes ${ }^{10}$. Otro aspecto a destacar es el corto período de tiempo entre el desarrollo del linfedema y la aparición del angiosarcoma, ya que la literatura cita una media de 10 años para el desarrollo de esta complicación ${ }^{11,12}$. La evolución es habitualmente agresiva, con rápido crecimiento de las lesiones en lo locorregional y tendencia a dar metástasis a distancia en corto plazo ${ }^{6}$ como se observó en el caso relatado que debutó con metástasis pulmonares. La media de sobrevida es de 19 meses $^{13}$ con rango de 8,5 a $13,6 \%$ a 5 años ${ }^{9}$. Una revisión de 160 casos, no halló diferencia significativa en la supervivencia entre los inicialmente tratados con amputación del miembro en comparación con los tratados con escisión local de las lesiones.
Tanto la amputación como la escisión mejoraron la sobrevida en comparación a la radioterapia o quimioterapia ${ }^{14}$. La presencia de metástasis pulmonares en el caso relatado, alejaron la opción quirúrgica como tratamiento posible.

El angiosarcoma desarrollado sobre un miembro con linfedema crónico o síndrome de StewartTreves es una complicación infrecuente pero de rápida y agresiva evolutividad. Hasta el momento no existe tratamiento curativo, presentando los afectados importante morbilidad y escasa expectativa de vida. La presencia de lesiones inhabituales en un miembro con linfedema debe conducir a la realización de una biopsia con el objetivo de despistar precozmente esta complicación. Deben optimizarse las medidas médicas para prevenir el linfedema de miembros cuando existen causas secundarias tratables, evitando así la elevada morbilidad que genera y las complicaciones mortales como la descrita.

Agradecimientos: A la Cátedra de Dermatología del Hospital de Clínicas, Facultad de Medicina, Universidad de la República por su aporte en lo que refiere a diagnóstico clínico e histopatológico.

\section{Referencias}

1. Morgan M, Swann M, Somach S, Eng W, Smoller B. Cutaneous angiosarcoma: A case series with prognostic correlation. J Am Acad Dermatol 2004; 50: 867-74.

2. Rodrígues E, Filizola E, Valdeci de Almeida F, Carvalho 
L, Ferreira S, Aguiar R. Síndrome de Stewart Treves: Relato de Caso. RBGO 2003; 25: 211-4.

3. Kerchner K, Fleischer A, Yosipovitch G. Lower extremity lymphedema. J Am Acad Dermatol 2008; 59: 324-31.

4. Clements WD, Kirk SJ, Spence RA. A rare late complication of breast cancer treatment. Br J Clin Pract 1993; 47: 219-20.

5. Komorowski AL, Wysocki WM, Mitus J. Angiosarcoma in a chroniclly lymphedematous leg: an unusual presentation of Stewart-Treves syndrome. South Med J 2003; 96: 807-8.

6. Maldonado N, López C, Sánchez J, Rodríguez A, Fernández F, Martínez J, et al. Síndrome de Stewart-Treves: linfangiosarcoma en linfedema crónico postmastectomía. Angiología 2002; 54 (6): 467-71.

7. Bisceglia M, Attino V, D'Addetta C, Murgo R, Fletcher CD. La sindrome de Stewart-Treves in fase precoce: presentazione di due casi e revisione della letteratura. Pathologica 1996; 88: 483-90.

8. De Vita VT, Hellman S, Rosenberg AS. Sarcomas of the soft tissues and bone. In: De Vita VT, Hellman S, Rosenberg AS, editors. Cancer: principles and practice of oncology. 6th ed. Philadelphia: Lippincott Williams \& Wilkins; 2001. p. 1841-83.

9. Damjanov I, Linder J. Soft - tissue tumors. In: Damjanov I, Linder J, editors. Anderson's Pathology. 10th ed. St Louis: Mosby; 1996. p. 2500.

10. Janse AJ, van Coevorden F, Peterse H, Keus RB, Dongen JA van. Lymphedema-induced lymphangiosarcoma. Er J Surg Oncol 1995; 21: 155-8.

11. Woodward AH, Ivins JC, Soule EH. Lymphangiosarcoma arising in chronic lymphedematous extremities. Cancer 1972; 30: 562-71.

12. Chung KC, Kim HJ, Jeffers LL. Lymphangiosarcoma (Stewart-Treves syndrome) in postmastectomy patients. J Hand Surg 2000; 25: 1163-8.

13. Stewart NJ, Pritchard DJ, Nascimento AG, Kang YK. Lymphangiosarcoma following mastectomy. Clin Orthop Relat Res 1995; 320: 135-41.

14. Grobmyer SR, Daly JM, Glorzbach RE, Grobmyer AJ. Role of surgery in postmastectomy extremity angiosarcoma (Stewart-Treves Syndrome). J Surg Oncol 2000; 73: 182-8. 